\title{
VIOLENCIA INTRAFAMILIAR CONTRA \\ A PESSOA IDOSA EM ÁREA URBANA COM SUPORTE DE PROTEÇÃO SOCIAL E DE SAÚDE
}

\author{
Maria de Nazaré de Souza Ribeiro' \\ Fátima Helena do Espírito Santo² \\ Cleisiane Xavier Diniz ${ }^{3}$ \\ Selma Lira Ribeiro ${ }^{4}$ \\ Vanusa do Nascimento ${ }^{5}$ \\ Euler Esteves Ribeiro ${ }^{6}$
}

1 Graduada em Enfermagem. Doutora em Ciências. Professora Adjunta da Universidade do Estado do Amazonas, vinculada ao Curso de Enfermagem e Departamento Científico de Enfermagem Gerontológica da Associação Brasileira de Enfermagem-ABEn-AM. Pós-doutoranda do Programa Acadêmico em Ciências do Cuidado - PACCS/UFF. E-mail: mnribeiro2@gmail.com.

2 Graduada em Enfermagem. Doutora em Enfermagem. Professora Associada da Universidade Federal Fluminense, Escola de Enfermagem Aurora de Afonso Costa, vinculada ao Programa Acadêmico em Ciências do Cuidado - PACCS/UFF e Departamento de Enfermagem Médico Cirúrgica. Coordenadora do Projeto Cuidar e Espaço Saúde: ambulatório de práticas alternativas para idosos (UFFESPA/UFF). Coordenadora do Curso de Pós-Graduaçao em Enfermagem Gerontológica. E-mail: fatahelens@gmail.com.

3 Graduada em Enfermagem. Doutora em Ciências. Professora Adjunta da Universidade do Estado do Amazonas, vinculada ao Curso de Enfermagem e Departamento Científico de Enfermagem Gerontológica da Associação Brasileira de Enfermagem-ABEn-AM. Pós-doutoranda do Programa Acadêmico em Ciências do Cuidado - PACCS/UFF. E-mail: cxdiniz@gmail.com.

4 Graduada em Serviço Social. Especialista em Gerontologia e Saúde do Idoso. Coordenadora da Pastoral da Pessoa Idosa. E-mail: s-lira@hotmail.com.

5 Graduada em Enfermagem. Mestre em Gerontologia. Coordenadora da Policlínica da Fundação Universidade Aberta da Terceira Idade - FuNATI. E-mail: vanusanascimento@gmail.com.

6 Graduado em Medicina. Doutor em Medicina e Ciências da Saúde. Professor Adjunto da Universidade do Estado do Amazonas. Reitor da Fundação Universidade Aberta da Terceira Idade - FuNATI. E-mail: unatieuler@gmail.com. 
resumo

Este estudo tem como objetivo estimar a prevalência da violência contra a pessoa idosa e investigar como ela se apresenta em área urbana com suporte de serviços de proteção social e de saúde destinados ao atendimento do idoso. Pesquisa quantitativa, transversal e descritiva realizada com 380 idosos residentes na zona sul da cidade de Manaus, área mais equipada com serviços de proteção social e de saúde para idosos. Foi calculada uma margem de erro de 5\% e Coeficiente de Confiança de 95\%. Utilizou-se Instrumento de Avaliação de Violência e Maus-Tratos Contra a Pessoa Idosa usado pelo Ministério da Saúde nos Cadernos de Atenção Básica. A prevalência de violência foi de 30,3\% e ultrapassou a média de muitos estudos brasileiros. A violência psicológica foi a mais prevalente, com taxa acima da média nacional, seguida da financeira e da física. As variáveis "idade", "renda familiar", "violência financeira" e "violência física" apresentaram forte associação ( $p<0,05)$. O número ampliado de unidades de acolhimento e notificação de violência não garantiu prevalência significantemente menor em relação à taxa nacional, indicando que o problema possa estar nas relações intrafamiliares conflituosas. Sugerem-se estudos que possam identificar a disfuncionalidade familiar como preditora de violência contra pessoa idosa e uma maior atuação das instituições que lidam com a problemática, na promoção de uma cultura de paz no âmbito da família e da comunidade.

palavras-chave

Envelhecimento. Violência. Violência Doméstica. Condições Sociais. Relações Familiares.

A população brasileira tem passado por um acelerado processo de envelhecimento em um contexto de expressiva desigualdade social, campo fértil para a exposição de idosos à violência, principalmente quando eles se encontram em condição de dependência para os cuidados básicos de vida, baixo nível socioeconômico, despreparo da família e dificuldades de acesso aos serviços de proteção social e de saúde (BELISÁRIO et al., 2018; SANTOS et al., 2019; SANTOS et al., 2020). 
O tema da violência contra a pessoa idosa ainda é um tabu na sociedade contemporânea, pois ocorre principalmente na esfera familiar, com os filhos e cônjuges apontados como principais agressores. O silêncio e a cumplicidade tornam o fenômeno da violência intrafamiliar contra a pessoa idosa um desafio para as políticas públicas e sociais (MALLET et al., 2016; ALARCON et al., 2019). O Ministério da Saúde define violência como eventos resultantes de atos intencionais, que compreendem a agressão, o homicídio, o abuso sexual, a negligência ou abandono, o abuso psicológico, a lesão autoprovocada, entre outras, que são passíveis de prevenção (BRASIL, 2015). A World Health Organization (WHO, 2002) conceitua violência contra pessoas idosas como ato único ou repetido ou ausência de atenção necessária, que cause danos/ sofrimento ou promova angústia para a pessoa idosa. Com relação à violência intrafamiliar ela é definida como toda ação ou omissão que interfira no bem-estar, na integridade física, psicológica ou na liberdade e no direito ao pleno desenvolvimento de outro familiar. Pode ocorrer dentro ou fora do domicilio por algum familiar, incluindo aqueles que assumem função parental, mesmo sem laços consanguíneos, e em relação de poder à outra. Essa violência não se refere apenas ao ambiente físico onde ocorre, mas também nas relações em que se constrói e efetua (BRASIL, 2001).

No Brasil, os tipos de violência contra a pessoa idosa mais prevalentes são a psicológica, a financeira e a física (LEE et al., 2018; CHIRIBOGA; BARREZUETA; AGUIRRE, 2018; ALARCON et al., 2019; BARROS et al., 2019; MAIA et al., 2019; SANTOS et al., 2019; SAMPAIO; SAMPAIO; VILELA, 2019). Define-se violência psicológica todo tipo de humilhação, rejeição, discriminação, desrespeito, cobrança excessiva, depreciação, punições e uso do idoso para atender às necessidades de outra pessoa. Normalmente, ocorre em forma de gritos, insultos, ameaças e constrangimentos, que levam à perda da dignidade, com danos à autoestima, à identidade ou ao desenvolvimento da pessoa e conduzem ao isolamento social. A violência financeira, também conhecida como econômica ou patrimonial, implica em dano, subtração, destruição ou retenção de objetos, documentos pessoais, bens e valores da pessoa idosa. Consiste, ainda, na exploração ilegal ou no uso não consentido de seus recursos financeiros e patrimoniais. A interdição de pessoa idosa sem perda de autonomia e/ou independência também é considerada violência financeira. A violência física ocorre quando alguém que se encontra em relação de poder desigual a outra tenta, por meio do uso da força física ou de algum tipo de arma, causar dano não acidental, com ou sem lesões externas, internas ou ambas (BRASIL, 2013).

As pessoas idosas, principalmente aquelas com comorbidades, dependência física, déficits cognitivos, alterações de sono, incontinência e dificuldades 
de locomoção, são as mais necessitadas de cuidados. Elas tendem a depositar sua confiança no membro familiar cuidador que, perante as dificuldades de prover as condições necessárias de cuidado, pode tornar-se o violador de seus direitos, provocando situações de violência e maus-tratos (DIEL; BARBIANI, 2018; ALARCON et al., 2019; COLUSSI et al., 2019). Embora o envelhecimento seja uma realidade incontestável, alcançar o estado de direito pleno para as pessoas idosas ainda é realidade difícil de ser vivenciada. A falta de uma agenda política nacional, que discuta a efetivação dos direitos das pessoas idosas já contidos em inúmeros documentos oficiais brasileiros, mas ábdito da cidadania de fato e de direito, converge para tal cenário (BRASIL, 1994; 2002; 2003; 2005; 2006a; 2015).

Em 1975, o tema da violência contra o idoso entrou oficialmente nas agendas internacionais de pesquisas e programas de ação na área da gerontologia, sendo alavancado com a fundação International Network for the Prevention of Elder Abuse (INPEA), uma rede de pesquisa e subsídio criada em 1997, com a finalidade de formulação de políticas para o idoso e o combate à violência. A Declaração de Madrid, de 2002, também tratou como prioridade a prevenção da violência contra o idoso. No Brasil, o tema ganhou reforço em 2011, com a obrigatoriedade da notificação da violência contra o idoso pelos serviços de saúde por meio do Sistema de Vigilância de Violências e Acidentes (VIVA) (MINAYO et al., 2018; ALARCON et al., 2019; BRASIL, 2019a). Com a Política Nacional de Saúde da Pessoa Idosa (PNSPI), aprovada pela portaria 2.528, de 19 de outubro de 2006, a rede de suporte de saúde, social, comunitária e familiar tornou-se um fator importante de redução de vulnerabilidade para ocorrências de violência (MINAYO et al., 2018).

$\mathrm{O}$ apoio social e de saúde que as redes concedem remete a mecanismos recíprocos de ajuda, no compartilhamento de informações, auxílio em momentos de crise e favorecimento de espaços de interação social na comunidade, gerando aumento de resiliência, da autoconfiança e da satisfação com a vida (SANT'ANA; D'ELBOUX, 2019; DOEKHIE et al., 2020; ARANEDA; LOWICK-RUSSELL, 2020).

Assim, é de se esperar que comunidades cujo suporte social e de saúde possua maior número de serviços de acolhimento ao idoso e à sua família destinadas à saúde ou ao atendimento de suas necessidades psicossociais, culturais e espirituais, dentre outras, apresentem índices de prevalência mais baixos de violência intrafamiliar e outras violências, uma vez que esses equipamentos, de forma direta ou indireta, ampliam as condições de enfrentamento à exclusão social, dão suporte à família e promovem maior qualidade de vida e proteção ao idoso (WANDERBROOCKE, 2017; SANT'ANA; D'ELBOUX, 2019). 
Dessa forma, aumentar a qualidade e a quantidade da rede de apoio social à família e ao idoso pode ser uma estratégia efetiva para proporcionar um envelhecimento bem-sucedido e livre de violência (DIEL; BARBIANI, 2018; PIMENTEL et al., 2019; MAIA et al., 2019). A identificação sistemática de pessoas idosas em situação de violência ou em contextos de vida que promovam um maior risco para sofrê-la é lacuna nas atuais práticas de atenção ao idoso no Brasil, conforme as estatísticas subestimadas dessa violência (SANTOS et al., 2018; MINAYO et al., 2018; BOLSONI et al., 2016).

O Relatório Mundial Sobre a Prevenção da Violência mostra que somente $17 \%$ dos países avaliados realizaram pesquisas nacionais de base populacional sobre violência contra pessoas idosas (WHO, 2014), e o Brasil não está entre eles. Isso significa que, na realidade brasileira, as estratégias para conter a violência são criadas sem que a problemática tenha sido amplamente estudada (SANTOS et al., 2020). Da mesma forma, não se encontram estudos que permitam identificar se, em comunidades com suporte de serviços de proteção social e de saúde destinados ao idoso, a violência contra a pessoa idosa mostra-se com padrões de prevalência diferenciada, principalmente aquela que acontece no ambiente familiar.

Assim, este estudo traçou as seguintes questões de pesquisa: Qual a prevalência da violência no grupo estudado? Em comunidades com suporte de serviços de proteção social e de saúde, a violência intrafamiliar praticada contra a pessoa idosa se apresenta com taxas significativamente mais baixas em relação à média nacional? O objetivo do estudo foi estimar a prevalência da violência contra a pessoa idosa e investigar como ela se apresenta em área urbana com suporte de serviços de proteção social e de saúde destinados ao atendimento do idoso. Este estudo não pretendeu avaliar a suficiência dos serviços em termos de qualidade e quantidade, tendo apenas considerado as instalações de suportes de saúde e social implantadas na comunidade.

Este estudo justifica-se pelo fato do rastreamento efetivo da violência contra a pessoa idosa, a partir do uso de ferramentas construídas e validadas para essa finalidade, poder ser um caminho inicial para que se descortine a problemática no ambiente familiar, com enfoque em seu dimensionamento, sua tipificação e sua localização. Possibilita também identificar fatores de risco, direcionando ações preventivas, norteando a implantação e o melhoramento dos serviços de proteção e redes assistenciais direcionadas à pessoa idosa e sua família, além de apontar para a necessidade de avaliação da eficácia das medidas protetivas ao idoso. 
Trata-se de estudo quantitativo, de natureza transversal e descritivo, oriundo do projeto intitulado Cartografia da violência intrafamiliar contra a pessoa idosa, que visa mapear a violência distribuída por zonas administrativas na cidade de Manaus (AM). A pesquisa foi aprovada pelo Comitê de Ética em Pesquisa da Universidade do Estado do Amazonas, sob parecer 3.173.698, conforme preconizado na resolução 466/12, do Ministério da Saúde, sobre pesquisas envolvendo seres humanos. Todos os participantes da pesquisa foram informados quanto aos objetivos e aos procedimentos de coleta de dados e esclarecidos quanto à assinatura do Termo de Consentimento Livre e Esclarecido (TCLE).

O estudo foi realizado na zona Sul da cidade de Manaus, por esta ser a com maior número de instalações de serviços sociais e de saúde públicos para atendimento de pessoas idosas, dos quais destacam-se: quatro Centros de Referência de Assistência Social (CRAS), dois Centros de Referência Especializado de Assistência Social (CREAS), uma Unidade da Fundação Universidade Aberta da Terceira Idade (FUNATI), um Centro de Atenção Integral à Melhor Idade (CAIMI), um Centro de Convivência da Família, oito Distritos Integrados de Polícia, uma Delegacia Especializada de Crime Contra Idoso (DECCI), uma Delegacia da Mulher, um Serviço de Pronto Atendimento, 16 Unidades Básicas de Saúde (UBS), um Hospital Geral, uma Instituição de Longa Permanência, uma unidade do Sesc, Secretaria Estadual de Assistência Social (SEAS), Secretaria de Estado de Justiça, Direitos Humanos e Cidadania, Secretaria Municipal da Mulher, Assistência Social e Cidadania (SEMASC), Secretaria Municipal de Educação (SEMED), Secretaria Estadual de Saúde (SUSAM), diversos grupos de idosos dentre outros serviços destinados à educação, cultura, espiritualidade etc.

A estimativa populacional da zona sul da cidade de Manaus para o ano de 2019 foi de 338.875 habitantes (IBGE, 2018). Baseado no universo da população residente nessa zona, realizou-se um cálculo amostral que totalizou 380 idosos, utilizando-se margem de erro amostral tolerável de $5 \%$ e coeficiente de segurança de $95 \%$. A amostra foi obtida de forma aleatória, mediante convocatória, por meio de documento expresso, contendo objetivo e forma de execução do projeto, além de reuniões com lideranças e grupos de idosos para participação na pesquisa nos centros comunitários, igrejas, associações e demais serviços de atendimento à pessoa idosa, localizados na zona sul da cidade.

Os critérios de elegibilidade para a participação da pesquisa foram: idosos com idade $\geq 60$ anos, residentes na zona Sul da cidade de Manaus, com 
capacidade para compreender e responder as perguntas do estudo e não ser autodeclarado indígena. Foi considerada somente uma pessoa por domicílio - preferencialmente, o membro mais velho -, que conseguisse se expressar e participar da pesquisa. Foram excluídos os indivíduos com alguma manifestação de sofrimento psíquico durante as entrevistas e/ou dificuldade de compreensão das perguntas. A coleta de dados ocorreu no período de agosto de 2019 a janeiro de 2020, e as entrevistas aconteceram de forma individualizada, em salas privativas, de acesso restrito nos centros de convivência, Unidades Básicas de Saúde, centros comunitários e igrejas. Todas as entrevistas foram realizadas sem a presença do acompanhante do idoso, para que fosse garantida sua liberdade de fala e segurança.

Para a identificação do perfil do idoso, utilizou-se um questionário contendo variáveis de gênero, renda, individual, participação econômica na família, autodeclaração de saúde, limitação física, presença de cuidador e relação com o cuidador. Para os dados relacionados à violência, utilizou-se o Instrumento Avaliação de Violência e Maus-tratos Contra a Pessoa Idosa, desenvolvido em Porto Rico e adotado pelo Ministério da Saúde nos Cadernos de Atenção Básica. Nele, investiga-se a violência física, psicológica e abuso financeiro. Uma resposta positiva a um dos itens caracteriza presença de violência contra a pessoa idosa (BRASIL, 2006b).

O instrumento foi replicado na plataforma Survey Monkey, a qual esta alimentou automaticamente o banco de dados. A plataforma Survey Monkey promove desenvolvimento de pesquisas on-line com recurso de salvamento de dados (informações) na nuvem, ou seja, cria um banco de dados on-line de acesso remoto, provê pesquisas personalizáveis em uma suíte de programas que inclui análise de dados, seleção de amostras, eliminação de vieses e ferramentas de representação de dados. Neste estudo, Survey Monkey permitiu que o instrumento fosse acessado a partir de tabletes para a realização das entrevistas. Para a análise dos dados, foi utilizado o software do programa Epi-Info ${ }^{\mathrm{TM}}$ versão 1 para Windows que é desenvolvido e distribuído gratuitamente pelo Centers for Disease Control and Prevention (CDC), adotando-se nível de significância fixado nos testes estatísticos de 5\% e Coeficiente de Confiança de 95\%.

Os dados foram apresentados em tabelas, onde calcularam-se as frequências absolutas simples e relativas para os dados categóricos. Na análise dos dados quantitativos, quando aceita a hipótese de normalidade por meio do teste de Shapiro-Wilk, foram calculadas a média e o desvio padrão (DP), no entanto, quando rejeitada a hipótese de normalidade, foram calculadas a mediana e os quartis $Q_{1(25 \%)}$ e $Q_{3(75 \%)}$. Já na análise dos dados categóricos, foi aplicado o teste qui-quadrado de Pearson, e, na impossibilidade de aplicar o 
teste de Pearson em tabelas 2x2, foi aplicado o teste exato de Fisher (VIEIRA, 2018). Na comparação das medianas, foi aplicado o teste não paramétrico de Mann-Whitney.

\section{Resultados e Discussão}

Na avaliação do perfil sociodemográfico dos idosos da presente pesquisa, observou-se que 76\% eram mulheres, na faixa de 69 anos (Tabela 1), semelhante a vários outros estudos, nos quais houve predominância do sexo feminino (GUIMARÃES et al, 2016; BARROS et al., 2019; LLANO et al., 2019; SILVA et al., 2019), com faixa etária aproximada. O estudo aconteceu fora do domicílio da pessoa idosa, de modo que as que participaram tinham condição melhor de mobilidade para comparecerem à chamada para participação na pesquisa nos diversos espaços de atendimento. Assim, a faixa acima de 80 anos tornou-se mal representada na pesquisa.

A maior parte dos idosos ganhava menos que dois salários mínimos, dividiam as despesas familiares e sustentava sua família. No Brasil, cerca de 12 milhões de famílias vive da aposentadoria ou benefício recebido pelos idosos e, mesmo em famílias nas quais outros membros trabalham, a renda fixa do idoso é a garantia da manutenção das despesas básicas. Dessa forma, a caracterização do idoso como um fardo para a família imposto a muitos idosos não deveria corresponder à realidade brasileira (BARROS et al., 2019; SAMPAIO; SAMPAIO; VILELA, 2019). Num estudo de Braga, Farias e Pimenta (2019), 64\% dos idosos homens e 77\% das idosas mulheres sustentavam ou dividiam sua renda com seus familiares. Nas novas configurações familiares, idosos independentes, além de ajudarem nas finanças da casa, ainda auxiliam filhos e netos, sendo-lhes atribuído novas responsabilidades sociais, até como cuidadores, quando normalmente os papéis são considerados inversos (SILVA et al., 2015). Porém, o fato de estarem contribuindo financeiramente e com afazeres domésticos não garante a eles coparticipação nas decisões familiares. 
Tabela 1 - Distribuição dos dados demográficos, de saúde e de relação familiar de pessoas idosas amostradas na zona sul da cidade de Manaus (AM), Brasil, 2020.

\begin{tabular}{|c|c|}
\hline Variáveis $(n=380)$ & $\mathrm{n}(\%)$ \\
\hline \multicolumn{2}{|l|}{ Gênero } \\
\hline Feminino & $289(76)$ \\
\hline Masculino & $91(24)$ \\
\hline \multicolumn{2}{|c|}{$\begin{array}{l}\text { Q1 - mediana - Q3 = } 64-69-75 \\
\text { Amplitude }=60-98\end{array}$} \\
\hline \multicolumn{2}{|l|}{ Faixa etária } \\
\hline $60-65$ & $100(26,3)$ \\
\hline $65-70$ & $95(25)$ \\
\hline $70-75$ & $84(22)$ \\
\hline $75-80$ & $52(13,7)$ \\
\hline $80>$ & 49 (13) \\
\hline \multicolumn{2}{|c|}{ Renda individual (salários mínimos) } \\
\hline Nenhuma & $10(2,6)$ \\
\hline$\leq 1$ & $128(33,7)$ \\
\hline $1-2$ & $149(39,2)$ \\
\hline $2-5$ & $69(18,2)$ \\
\hline $5-10$ & $20(5,3)$ \\
\hline$>10$ & $4(1,0)$ \\
\hline
\end{tabular}

Participação econômica na família

$\begin{array}{ll}\text { Divide as despesas } & 234(61,6)\end{array}$

Único (a) responsável $115(30,3)$

$\begin{array}{ll}\text { Sem participação } & 29(7,6)\end{array}$

Não sabe $\quad 2(0,5)$

\section{Em geral sua saúde está}

Ótima

Boa

Ruim 


\begin{tabular}{ll}
\hline Variáveis $(\mathrm{n}=380)$ & $\mathrm{n}(\%)$ \\
\hline Possui alguma limitação física & $94(24,7)$ \\
Possui cuidador & $115(30,3)$ \\
Relacionamento com o cuidador $(\mathrm{n}=115)$ & \\
Ótimo & $50(43,5)$ \\
Bom & $62(53,9)$ \\
Regular & $3(2,6)$ \\
\hline
\end{tabular}

Fonte: Pesquisa de campo, 2019/2020.

Quanto à autoavaliação de saúde, $66 \%$ dos participantes consideraram sua condição boa e realizaram a maioria de suas atividades sozinhos. Contudo, 24,7\% relataram possuir alguma limitação física, necessitando da ajuda de algum cuidador. A autoavaliação de saúde expressa a percepção da pessoa idosa, considerando minimamente as dimensões biológica e psicossocial de sua condição de saúde. Mais da metade dos idosos classificou sua saúde autopercebida como boa, divergindo do estudo de Barros et al. (2019), em que a maioria atribuiu valores insatisfatórios à própria saúde. Pesquisas têm demonstrado que pessoas idosas que autodeclaram uma condição de saúde escassa ou pobre têm riscos de mortalidade substancialmente mais altos que idosos com percepções positivas de seu estado de saúde (SANTOS et al., 2018; BARROS et al., 2019).

$\mathrm{Na}$ autoavaliação de boa saúde, a maioria dos idosos também declarou pouca limitação física para o autocuidado e sem necessidade de cuidador. Somente 30,3\% disseram ter um cuidador, que, em geral, é um familiar, reconhecendo existir um relacionamento entre ótimo (43,5\%) e bom (53,9\%) entre eles. Por outro lado, pesquisas apontam que a convivência entre idosos e seu cuidador familiar é quase sempre permeada de conflitos decorrentes da divergência de ideias, das dificuldades financeiras para manutenção das condições básicas, da falta de espaço físico e de divórcios que propiciam a volta dos filhos à casa dos pais, aumentando a dependência financeira e emocional dos primeiros, além de outras condições inerentes ao cuidador, que podem gerar situações de violência intrafamiliar (SANTOS et al., 2018; SAMPAIO; SAMPAIO; VILELA, 2019)

No grupo de idosos estudados, a prevalência geral da violência foi de $32,7 \%$, ocorrida com 167 idosos da amostra, sendo possível identificar três 
tipos de violência: a psicológica, a financeira e a física. Destacando-se apenas as respostas afirmativas para ocorrência da violência no último ano, observa-se que a violência psicológica alcançou maior prevalência (29,5\%), seguida da financeira $(10,3 \%)$ e física $(4,2 \%)$ (Tabela 2$)$, corroborando os estudos de Lopes et al. (2018), no qual as violências psicológica, física e financeira também apresentaram os maiores índices. Em vários estudos, a violência financeira apresenta-se como mais prevalente em homens enquanto a psicológica ocorre mais entre as mulheres (LEE et al., 2018; CHIRIBOGA; BARREZUETA; AGUIRRE, 2018; ALARCON et al., 2019; MAIA et al., 2019). A violência física foi a menos relatada entre os participantes, com prevalência de $4,2 \%$, divergindo de alguns estudos cujos níveis são variados (entre 0,3 e 58,9\%) (BOLSONI et al., 2016; SANTOS et al., 2019).

Tabela 2 - Prevalência da violência contra a pessoa idosa amostradas na zona sul da cidade de Manaus (AM), Brasil, 2020.

\begin{tabular}{lll}
\hline Tipo de violência $(\mathrm{n}=167)$ & Violência $\mathrm{n}(\%)$ & IC95\% \\
\hline Psicológica & $112(29,5)$ & $17,7-26,0$ \\
Financeira & $39(10,3)$ & $5,2-10,4$ \\
Física & $16(4,2)$ & $2,0-5,8$ \\
\hline
\end{tabular}

Fonte: Pesquisa de campo 2019/2020.

IC95\% = intervalo de confiança ao nível de 95\%.

A ampliação do número de unidades de acolhimento e notificação de violência, bem como o fortalecimento da rede de atenção e de proteção integral às pessoas em situação de violência, permanece como uma estratégia fundamental de combate ao problema, porém, se a violência intrafamiliar continua a manter níveis de prevalência elevados é preciso que os membros da família busquem modificar as dinâmicas de poder/afeto entre si, principalmente em famílias em que estão presentes relações de subordinação-dominação, com os idosos assumindo a posição de subordinação, cada vez mais expostos à violência de toda natureza. Na estratificação da violência psicológica, 17,6\% relataram serem vítimas de gritos por pessoas próximas, sem nenhuma razão, $9 \%$ foram tratados de forma pejorativa (apelidos), e 2,9\% sofreram ameaças por se oporem à vontade de outros (Tabela 3). A violência psicológica é expressada por atitudes de agressões verbais ou gestuais, humilhação, insultos e chantagem, que provocam sentimentos de medo, raiva, choro e desvalorização. 
Dentre as várias tipologias da violência contra a pessoa idosa, estudos apontam a psicológica como a mais comum no Brasil, relacionada a ações com intenção de causar dano emocional, angústia e aflição. Sua prevalência varia de 19\% a 69,6\%, dependendo da população estudada (GUIMARÃES et al., 2016; BOLSONI et al., 2016; DIEL; BARBIANI, 2018; BARROS et al., 2019; MAIA et al., 2019).

Tabela 3 - Frequência das variáveis do da avaliação de violência e maus-tratos contra a pessoa idosa aplicado aos idosos amostrados na zona sul da cidade de Manaus (AM), 2020.

\begin{tabular}{|c|c|c|c|c|c|c|}
\hline \multirow{2}{*}{ Variáveis $(n=167)$} & \multirow{2}{*}{$\underset{\mathrm{n}(\%)}{\operatorname{Sim}}$} & \multicolumn{5}{|c|}{ Com que frequência (\%) } \\
\hline & & 1 & 2 & 3 & 4 & $5>$ \\
\hline \multicolumn{7}{|l|}{ Violência psicológica } \\
\hline $\begin{array}{l}\text { No último ano, alguma das pessoas que } \\
\text { o(a) rodeiam tem gritado com o(a) Sr.(a) sem } \\
\text { razão? }\end{array}$ & 17,6 & 7,5 & 17,9 & 7,5 & 14,9 & 52,2 \\
\hline $\begin{array}{l}\text { No último ano, alguma das pessoas que o(a) } \\
\text { rodeiam tem chamado por algum nome ou } \\
\text { apelido que o(a) Sr(a) não goste? }\end{array}$ & 9,0 & 15,5 & 9,1 & 9,1 & 12,1 & 54,6 \\
\hline $\begin{array}{l}\text { No último ano, alguma das pessoas que o(a) } \\
\text { rodeiam tem ameaçado por não fazer o que } \\
\text { eles querem que o(a) Sr.(a) faça? }\end{array}$ & 2,9 & 27,3 & 18,2 & - & 27,3 & 27,3 \\
\hline \multicolumn{7}{|l|}{ Violência financeira } \\
\hline $\begin{array}{l}\text { No último ano, alguma das pessoas que } \\
\text { o(a) rodeiam tem usado ou manejado seu } \\
\text { dinheiro sem seguir suas instruções? }\end{array}$ & 4,2 & 6,2 & - & 12,5 & 12,5 & 68,8 \\
\hline $\begin{array}{l}\text { No último ano, alguma das pessoas que o } \\
\text { rodeiam tem roubado seu dinheiro ou algum } \\
\text { de seus pertences? }\end{array}$ & 6,1 & 4,3 & - & 8,7 & - & 87,0 \\
\hline \multicolumn{7}{|l|}{ Violência física } \\
\hline $\begin{array}{l}\text { No último ano, alguma das pessoas que } \\
\text { o(a) rodeiam tem golpeado, batido ou } \\
\text { esbofeteado? }\end{array}$ & 1,6 & 16,7 & 16,7 & - & 16,7 & 50,0 \\
\hline $\begin{array}{l}\text { No último ano, alguma das pessoas que o } \\
\text { rodeiam o(a) tem sacudido ou segurado de } \\
\text { forma intimidadora ou ameaçadora? }\end{array}$ & 2,6 & - & 20,0 & & 10,0 & 70,0 \\
\hline
\end{tabular}

Fonte: Pesquisa de campo, 2019/2020.

1: todos os dias da semana; 2: 2 ou 3 vezes na semana; 3: 1 vez na semana; 4: 2 a 3 vezes ao mês; 5: 1 vez ao mês ou menos. 
Quanto à violência financeira, 6,1\% foram vítimas de roubo de dinheiro ou usurpação de bens, e 4,2\% afirmaram que pessoas próximas usaram seu dinheiro indevidamente ou sem seu consentimento. A violência financeira alcançou o segundo maior nível de prevalência, da mesma forma que no estudo de Barros et al. (2019) e Bolsoni et al. (2016). Esse tipo de violência é apresentado no Estatuto do Idoso (BRASIL, 2008) como crime, caracterizado por apropriação ou desvio de bens, proventos, pensão ou outro rendimento. O Estatuto do Idoso declara ainda que reter o cartão de conta bancária relativa a proventos, benefícios ou pensão do idoso, ou outro documento, com objetivo de assegurar recebimento ou ressarcimento de dívida, caracteriza-se como violência financeira (BRASIL, 2008). No que se refere à violência física, apenas $2,6 \%$ relataram ter sido sacudidos ou segurados de forma intimidadora ou ameaçadora e 1,6\% foi golpeado, batido ou esbofeteado. A frequência maior na ocorrência de todos os tipos de violência foi uma vez por mês, alcançando mais de 50\% em todas as perguntas aplicadas. O Relatório 2019 do Disque Direitos Humanos mostra que as violações possuem continuidade temporal, com relato de ocorrências diárias e evidências de que as violações de direitos humanos não são fenômenos eventuais, mas recorrentes, pois a vítima é submetida a violações contínuas (BRASIL, 2019a).

Na Tabela 4, observa-se que a violência física apresentou forte associação com a renda $(\mathrm{p}<0,017)$ e a violência financeira com a faixa etária de $70 \mathrm{a}$ 83 anos ( $\mathrm{p}<0,001)$, indicando diferença estatística entre os grupos das variáveis de exposição ao nível de $5 \%$ de significância.

Tabela 4 - Comparação das variáveis em relação à tipologia da violência ao gênero, à renda familiar e à idade dos idosos amostrados na zona sul da cidade de Manaus (AM).

Tipo de violência

\begin{tabular}{|c|c|c|c|c|}
\hline Variáveis (n=380) & $\begin{array}{l}\text { Psicológica } \\
(\mathrm{n}=112)\end{array}$ & $\begin{array}{l}\text { Financeira } \\
(\mathrm{n}=39)\end{array}$ & $\begin{array}{l}\text { Física } \\
(n=16)\end{array}$ & Total \\
\hline \multicolumn{5}{|l|}{ Gênero } \\
\hline Feminino & $84(29,1)$ & $29(10,0)$ & $15(5,2)$ & $289(76,1)$ \\
\hline Masculino & $28(30,8)$ & $10(11,0)$ & $1(1,1)$ & $91(23,9)$ \\
\hline Valor de $\mathrm{p}^{*}$ & $0,325^{*}$ & $0,892^{*}$ & $0,317^{\mathrm{a}}$ & \\
\hline \multicolumn{5}{|l|}{ Renda familiar } \\
\hline$\leq 1 \mathrm{SM}$ & $42(30,4)$ & $16(11,6)$ & $10(7,2)$ & $138(36,3)$ \\
\hline$>1 \mathrm{SM}$ & $70(28,9)$ & $23(9,5)$ & $6(2,4)$ & $242(63,7)$ \\
\hline Valor de $\mathrm{p}^{*}$ & $0,565^{\star}$ & $0,454^{\star}$ & $0,017^{\mathrm{a}}$ & \\
\hline
\end{tabular}


Tipo de violência

\begin{tabular}{lcccc}
\cline { 2 - 4 } Variáveis $(\mathrm{n}=380)$ & $\begin{array}{c}\text { Psicológica } \\
(\mathrm{n}=112)\end{array}$ & $\begin{array}{c}\text { Financeira } \\
(\mathrm{n}=39)\end{array}$ & $\begin{array}{c}\text { Física } \\
(\mathrm{n}=16)\end{array}$ & Total \\
\hline Idade (Q1 - mediana - Q3) & & & \\
$\operatorname{Sim}$ & $63-68-75$ & $70-73-83$ & $62-68-80$ \\
Não & $65-69-75$ & $64-69-75$ & $64-69-75$ \\
Valor de $\mathrm{p}^{*}$ & $0,501^{\mathrm{b}}$ & $<0,001^{\mathrm{b}}$ & $0,929 \mathrm{~b}$ \\
\hline
\end{tabular}

Fonte: Pesquisa de campo, 2019/2020.

* Teste do qui-quadrado; a teste exato de Fisher; ${ }^{b}$ teste de Mann-Whitney. SM: salários mínimos.

Observa-se, ainda, que os homens sofreram mais violência psicológica e financeira, e as mulheres vivenciaram mais violência física. Embora os achados demonstrem que as maiores vítimas de abusos financeiros foram as pessoas idosas mais velhas, a literatura indica que idosos mais jovens conseguem perceber e relatar mais a violência sofrida, não especificamente porque são as maiores vítimas, mas porque podem se comunicar melhor (LEE et al., 2018; LOPES et al., 2018; ALARCON et al., 2019; MAIA et al., 2019). Na realidade brasileira, muitos idosos encontram-se endividados junto às financiadoras de crédito e agências bancárias, por conta de empréstimos para filhos e netos, tornando-se inadimplentes e sujeitos a uma nova violência financeira, por cobranças abusivas de juros (MELO et al., 2016). Segundo a WHO (WHO, 2012), anualmente, pelo menos 4 milhões de idosos passam por uma experiência de violência e maus-tratos, e cerca de 2.500 morrem em decorrência desse fenômeno. De cada caso de violência denunciada às autoridades, cinco não são reportados e cerca de $80 \%$ não são denunciados. Vários estudos realizados em diferentes países concluíram que qualquer pessoa idosa pode ser vítima da violência, independentemente da sua condição étnica, religião ou estado sociocultural (BOND; BUTLER, 2013; BURNETT; ACHENBAUM; MURPHY, 2014; SANTOS et al., 2020; DOEKHIE et al., 2020).

No Brasil, entre os anos de 2011 e 2017, houve aumento de 186\% no número de notificações de violências interpessoais (domésticas e comunitárias), com os principais acréscimos relativos às mulheres (194\%) e à faixa etária de 60 anos ou mais (261\%). Houve também aumento importante na cobertura do sistema de vigilância contínua, de 38,0\% de municípios notificantes, em 2011, para 71,6\%, em 2017 (BRASIL, 2019b). Dessa forma, os resultados deste estudo, realizado em área com suporte de serviços de proteção, pressupõem que a 
violência identificada pode estar sendo determinada por relações intrafamiliares conflituosas de subordinação e dominação e que se faz necessário investigar a disfuncionalidade familiar como preditora de violência contra a pessoa idosa, uma vez que o problema não parece estar no suporte externo.

Como limitação principal do estudo, considera-se a falta de participação das pessoas idosas dependentes, que não costumam frequentar atividades externas à sua residência por problemas de saúde física ou mental, mas que são as maiores vítimas apontadas nos inúmeros estudos investigativos.

\section{Conclusão}

O presente estudo buscou identificar a taxa de prevalência da violência contra a pessoa idosa e investigar se, em comunidade onde exista um considerável suporte de serviços de proteção social e de saúde destinados ao atendimento de pessoas idosas, a violência intrafamiliar contra o idoso apresenta-se em taxas significativamente mais baixas em relação à média nacional. Ratifica-se a violência psicológica como a mais prevalente, com taxa acima da média nacional; as violências financeira e física tiveram taxa mais baixa, porém próxima à média nacional. Quanto às variáveis dependentes, as que apresentaram forte evidência de estarem associadas a esse fenômeno da violência foram a renda financeira e a idade elevada.

O número ampliado de unidades de acolhimento e notificação de violência não garantiu uma prevalência significantemente menor em relação à taxa nacional, indicando que o problema possa estar nas relações intrafamiliares conflituosas. Ainda, a articulação contínua da rede de proteção às pessoas idosas em situação de violência ou risco deve continuar a buscara atenção integral e humanizada no âmbito das políticas de assistência social, saúde e sistema de proteção e garantia dos direitos da pessoa idosa e sua família, reduzindo os possíveis fatores de insuficiência familiar que levam à violência.

O contato direto com os participantes da pesquisa em ambiente fora do domicílio e em espaço privativo proporcionou a eles segurança para relatarem a violência sofrida, dando ao estudo melhor qualidade aos resultados. Destaca-se que, no Brasil, poucos são os inquéritos de base populacional, realizados diretamente com os idosos sobre violência, dificultando o conhecimento da magnitude do fenômeno em âmbito regional e nacional. Assim, este estudo pode servir de subsídio para a construção e o fortalecimento de políticas públicas para o enfrentamento da violência e sensor para as instituições que 

paz no âmbito da família e da comunidade.

\author{
INTERFAMILY VIOLENCE AGAINST ELDERLY \\ PEOPLE IN URBAN AREA WITH THE SUPPORT \\ OF SOCIAL AND HEALTH PROTECTION
}

\title{
abstract
}

The objective was to estimate the prevalence of violence against the elderly and to investigate how it presents itself in an urban area with support from social protection and health services for the elderly. Quantitative, cross-sectional and descriptive research carried out with 380 elderly people living in the southern area of the city of Manaus, an area more equipped with social protection and health services for the elderly. A 5\% margin of error and a 95\% confidence coefficient were calculated. An Instrument for the Assessment of Violence and Mistreatment against the Elderly, used by the Ministry of Health in the Primary Care Notebooks, was applied. The prevalence of violence was $30.3 \%$ and exceeded the average of many Brazilian studies. The psychological violence was the most prevalent with a rate above the national average, followed by financial and physical. The variables age, family income, financial and physical violence showed a strong association ( $\mathrm{p}<0.05)$. The increased number of reception units and notification of violence did not guarantee a significantly lower prevalence in relation to the national rate, indicating that the problem may be in conflicting interfamily relationships. It suggests studies that can identify family dysfunctionality as a predictor of violence against the elderly and the greater role of institutions that deal with the problem, in promoting a culture of peace within the family and community.

keywords

Aging. Violence. Domestic violence. Social Conditions. Family Relationship. 
ALARCON, Miriam Fernanda Sanches et al. Violence againstthe elderly: a documentary study. Revista da Rede de Enfermagem do Nordeste, Fortaleza, v. 20, e41450, 2019.

ARANEDA, Nelson Garcia; LOWICK-RUSSELL, Jenny. Elder abuse in Chile: evidence, legal regulations and immediate challenges. In: SHANKARDASS, Mala Kapur (ed.). International handbook of elder abuse and mistreatment. Singapore: Springer, 2020. p. 89-110.

BARROS, Renata Laíse de Moura et al. Violência doméstica contra idosos assistidos na atenção básica. Saúde Debate, Recife, v. 43, n. 122, p. 793-804, 2019.

BELISÁRIO, Mariane Santos et al. Cross-sectional study on the association between frailty and violence against community-dwelling elderly people in Brazil. São Paulo Medical Journal, São Paulo, v. 136, n. 1, p. 10-19, 2018.

BOLSONI, Carolina Carvalho et al. Prevalência de violência contra idosos e fatores associados, estudo de base populacional em Florianópolis, SC. Revista Brasileira de Geriatria e Gerontologia, Rio de Janeiro, v. 19, n. 4, p. 671-682, 2016.

BOND, Michael C.; BUTLER, Kenneth H. Elder abuse and neglect: definitions, epidemiology, and approaches to emergency department screening. Clinics Geriatric in Medicine, Amsterdã, v. 29, n. 1, p. 257-273, 2013.

BRAGA, Cilene Sebastiana da Conceição; FARIAS, Cimara de Lima; PIMENTA, Marisa Fernanda. Benefício de Prestação Continuada - Idoso: perfil e composição familiar dos assistidos pelo CRAS-Tapanã, em Belém-Pará. O Social em Questão, Rio de Janeiro, ano XXII, n. 43, p. 193-216, 2019.

BRASIL. Constituição da República Federativa do Brasil de 1988. Organização de Iracema Almeida Valverde et al. 2. ed. Rio de Janeiro: Expressão e Cultura, 2002.

BRASIL. Guia de Políticas, Programas e Projetos do Governo Federal. Compromisso Nacional para o Envelhecimento Ativo. Brasilia, DF: Secretaria de Direitos Humanos da Presidência da República, 2015.

BRASIL. Ministério da Mulher, da Família e dos Direitos Humanos. Ouvidoria Nacional de Direitos Humanos. Disque Direitos Humanos - Relatório 2019. Brasília, DF: Ministério da Mulher, da Família e dos Direitos Humanos, 2019a. Disponível em: http://www.cfess. org.br/arquivos/Disque100Relatorio.pdf. Acesso em: 17 jul. 2020.

BRASIL. Ministério da Saúde. Portaria n².258, de 19 de outubro de 2006. Aprova a Política Nacional de Saúde da Pessoa ldosa. Brasília, DF: Ministério da Saúde, 2006a. Disponível em: https://bvsms.saude.gov.br/bvs/saudelegis/gm/2006/prt2528_19_10_2006. html. Acesso em: 20 jul. 2020.

BRASIL. Ministério da Saúde. Secretaria de Atenção à Saúde. Departamento de Atenção Básica. Envelhecimento e saúde da pessoa idosa. Brasília, DF: Ministério da Saúde, 2006b. (Cadernos de Atenção Básica, n. 19). Disponível em: https://bvsms.saude.gov. br/bvs/publicacoes/evelhecimento_saude_pessoa_idosa.pdf. Acesso em: 17 jul. 2020.

BRASIL. Ministério da Saúde. Secretaria de Políticas de Saúde. Violência intrafamiliar: orientações para prática em serviço. Série Cadernos de Atenção Básica n. 8; Série A. Normas e Manuais Técnicos, n. 131. Brasília, DF: Ministério da Saúde, 2001. 96 p. Disponível em: http://bvsms.saude.gov.br/bvs/publicacoes/cd05_19.pdf. Acesso em: 13 ago. 2020. 
BRASIL. Ministério de Saúde. Secretaria Especial de Direitos Humanos da Presidência da República. Plano de Ação para o enfrentamento da violência contra a pessoa idosa. Brasilia, DF: Diário Oficial da União, 2005. Disponível em: http://www.observatorionacionaldoidoso.fiocruz.br/biblioteca/_manual/11.pdf. Acesso em: 20 jul. 2020.

BRASIL. Presidência da República. Casa Civil. Subchefia para Assuntos Jurídicos. Lei n. 8.842 de 04 de janeiro de 1994. Dispõe sobre a política nacional do idoso, cria o Conselho Nacional do Idoso e dá outras providências. Brasília, DF: Diário Oficial da União, 1994. Disponível em: http://www.planalto.gov.br/ccivil_03/leis/L8842.htm. Acesso em: 20 jul. 2020.

BRASIL. Presidência da República. Casa Civil. Subchefia para Assuntos Jurídicos. Lei n. 10.741, de $1^{\circ}$ de outubro de 2003. Dispõe sobre o Estatuto do Idoso e dá outras providências. Brasília, DF: Diário Oficial da União, 2003. Disponível em: http:// www.planalto.gov.br/ccivil_03/leis/2003/110.741.htm. Acesso em:20 jul. 2020.

BRASIL. Presidência da República. Casa Civil. Subchefia para Assuntos Jurídico. Lei n. 10.741, $1^{\circ}$ de outubro de 2003. Dispõe sobre Estatuto do Idoso e dá outras providências. Brasília, DF: Diário Oficial da União, 2008.

BRASIL. Rede Internacional de Educação de Técnicos em Saúde. Vigilância em saúde no Brasil 2003/2019: da criação da Secretaria de Vigilância em Saúde aos dias atuais. Brasília, DF: Boletim Epidemiológico, 2019b. Disponível em: http:// www.rets.epsjv.fiocruz.br/biblioteca/vigilancia-em-saude-no-brasil-20032019-da-criacao-da-secretaria-de-vigilancia-em-saude. Acesso em: 17 jul. 2020.

BRASIL. Secretaria de Direitos Humanos da Presidência da República. Manual de enfrentamento à violência contra a pessoa idosa. É possível prevenir. É necessário superar. Brasília, DF: Secretaria de Direitos Humanos da Presidência da República, 2013. Disponível em: https://craspsicologia.files.wordpress.com/2014/06/ violencia-contra-a-pessoa-idosa_miolo_para-web.pdf. Acesso em: 17 jul. 2020.

BURNETT, Jason; ACHENBAUM, W. Andrew; MURPHY, Kathleen Pace. Prevention and early identification of elder abuse. Clinics Geriatric in Medicine, Amsterdã, v. 30, n. 4, p. 743-759, 2014.

CHIRIBOGA, Efrén Viteri; BARREZUETA, Aida Elizabeth Terranova; AGUIRRE, Lazara Milagros Velis. Funcionalidad familiar y autoestima del adulto mayor, en situación de maltrato. Un estudio participativo comunitário. Revista Lasallista de Investigación, Antioquia, v. 15, n. 2, p. 300-314, 2018.

COLUSSI, Eliane Lucia et al. Percepções de idosos sobre envelhecimento e violência nas relações intrafamiliares. Revista Brasileira de Geriatria e Gerontolologia, Rio de Janeiro, v. 22, n. 4, e190034, 2019.

DIEL, Marciane; BARBIANI, Rosângela. Violência familiar contra a pessoa idosa: expressões do fenômeno e perspectivas para o seu enfrentamento. Revista Textos \& Contextos, Porto Alegre, v. 17, n. 2, p. 379-392, 2018.

DOEKHIE, Kirti et al. Elderly patients' decision-making embedded in the social context: a mixed-method analysis of subjective norms and social support. BioMed Central Geriatrics, v. 20, n. 53, p. 1-15, 2020.

GUIMARÃES, David Bernar Oliveira et al. Caracterização da pessoa idosa vítima de violência. Revista de Enfermagem da Universidade Federal de Pernambuco Online, Recife, v. 10, Supl. 3, p. 1343-1350, 2016

INSTITUTO BRASILEIRO DE GEOGRAFIA E ESTATÍSTICA (IBGE). Brasil em Síntese. Amazonas/Manaus/Panorama. IBGE, 2018. 
LEE, Jessica L. et al. Feasibility of intervention in elder self-neglecters: setting the stage for future research. Journal of Elder Abuse \& Neglect, London, v. 30, n. 3, p. 2232-2235, 2018.

LLANO, Patrícia Mirapalheta Pereira et al. Fatores associados a síndrome da fragilidade em idosos rurais. Revista Brasileira de Enfermagem, Brasília, DF, v. 72, Suppl. 2 p. 19-26, 2019

LOPES, Emmanuel Dias de Sousa et al. Maus-tratos a idosos no Brasil: uma revisão integrativa. Revista Brasileira de Geriatria e Gerontolologia, Rio de Janeiro, v. 21, n. 5 , p. 652-662, 2018.

MAIA, Paulo Henrique Silva et al. A ocorrência da violência em idosos e seus fatores associados. Revista Brasileira de Enfermagem, Brasília, DF, v. 72, Suppl. 2, p. 71-77, 2019.

MALLET, Sandra de Mendonça et al. Violência contra idosos: um grande desafio do envelhecimento. Revista Médica de Minas Gerais, Belo Horizonte, v. 26, Supl. 8 , p. S408-S413, 2016.

MELO, Natália Calais Vaz de et al. Arranjo domiciliar de idosos no Brasil: análises a partir da Pesquisa Nacional por Amostra de Domicílios (2009). Revista Brasileira de Geriatria e Gerontolologia, Rio de Janeiro, v. 19, n. 1, p. 139-151, 2016

MINAYO, Maria Cecilia de Souza et al. Institucionalização do tema da violência no SUS: avanços e desafios. Ciência \& Saúde Coletiva, Rio de Janeiro, v. 23, n. 6 , p. 2007-2016, 2018.

PIMENTEL, Maria Helena Pimentel et al. Importance of social network for the successful aging and health of the elderly. Journal of Aging \& Innovation, v. 8, n. 1, p. 68-84, 2019

SAMPAIO, Talita Santos Oliveira; SAMPAIO, Lucas Silveira; VILELA, Alba Benemérita Alves. Conteúdos e estrutura representacional sobre família para idosos em corresidência. Revista Ciência \& Saúde Coletiva, Rio de Janeiro, v. 24, n. 4, p. 1309-1316, 2019

SANT'ANA, Leila Auxiliadora José de; D' ELBOUX, Maria José. Comparação da rede de suporte social e a expectativa para o cuidado entre idosos em diferentes arranjos domiciliares. Revista Brasileira de Geriatria e Gerontolologia, Rio de Janeiro, v. 22, n. 3, p. e190012, 2019

SANTOS, Ana Maria Ribeiro et al. Violência econômico-financeira e patrimonial contra o idoso: estudo documental. Revista da Escola de Enfermagem da Universidade de São Paulo, São Paulo, v. 53, e03417, 2019

SANTOS, Maria Angélica Bezerra et al. Fatores associados à violência contra o idoso: uma revisão sistemática da literatura. Ciência \& Saúde Coletiva, Rio de Janeiro, v. 25, n. 6, p. 2153-2175, 2020.

SANTOS, Tamires Daros et al. Casos notificados de violência doméstica, sexual e/ou outras violências em idosos no município de Santa Maria - RS. Revista Saúde, Santa Maria, v. 44, n. 2, p. 1-12, 2018

SILVA, Doane Martins et al. Dynamics of intergenerational family relationships from the view point of elderly residents in the city of Jequié (Bahia), Brazil. Revista Ciência \& Saúde Coletiva, Rio de Janeiro, v. 20, n. 7, p. 2183-2191, 2015.

SILVA, Patrícia Aparecida Barbosa et al. Fatores associados a síndrome metabólica em idosos: estudo de base populacional. Revista Brasileira de Enfermagem, Brasília, DF, v. 72, Suppl. 2, p. 231-239, 2019.

VIEIRA, Sonia. Bioestatística, Tópicos Avançados. Rio de Janeiro. 4. ed. Rio de Janeiro: Elsevier, 2018. 308p. 
Data de Submissão: 11/05/2020

Data de Aprovação: 18/08/2020
WANDERBROOCKE, Ana Claudia Nunes de Souza. Idosas, rede social significativa e o enfrentamento da violência familiar. Estudos interdisciplinares sobre o Envelhecimento, Porto Alegre, v. 22, n. 1, p. 99-116, 2017.

WORLD HEALTH ORGANIZATION (WHO). Missing voices: views of older persons on elder abuse. Genebra: WHO, 2002. Disponível em: https://www.who.int/ageing/ publications/missing_voices/en/. Acesso em: 17 jul. 2020.

WORLD HEALTH ORGANIZATION (WHO). European report on preventing elder maltreatment. Genebra: WHO, 2012. Disponível em: https://apps.who.int/iris/handle/10665/107293. Acesso em: 17 jul. 2020.

WORLD HEALTH ORGANIZATION (WHO).Global status report on violence prevention 2014. Genebra: WHO, 2014. Disponível em: https://www.who.int/violence_injury_prevention/violence/status_report/2014/report/report/en. Acesso em: 17 jul. 2020. 